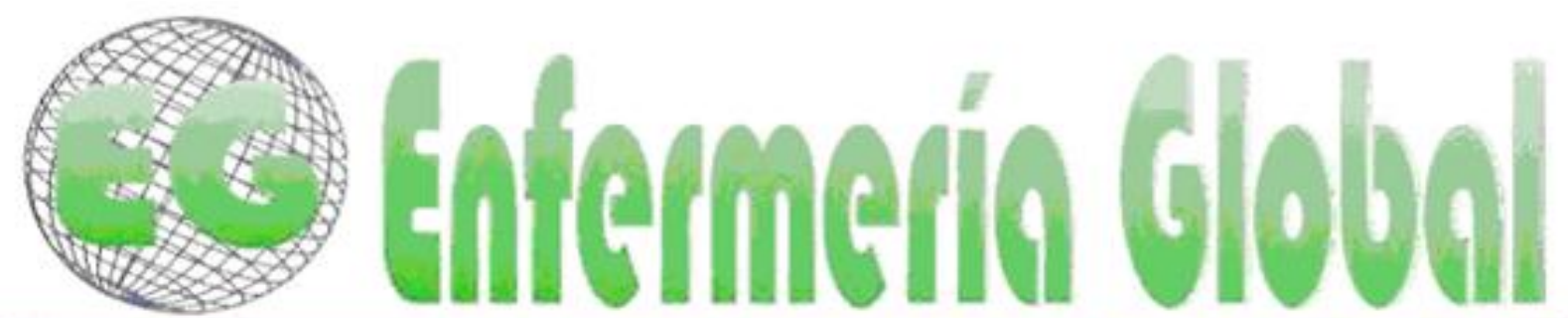

\title{
Cuidados de enfermería basados en evidencia y modelo de Betty Neuman, para controlar estresores del entorno que pueden ocasionar delirium en unidad de cuidados intensivos
}

Evidence -and Betty Neuman's model- based nursing care to prevent deilrium in the intensive care unit

\section{*Gómez Tovar, Luz Omaira **Díaz Suarez, Leticia ***Cortés Muñoz, Fabián

\begin{abstract}
*Magister en Enfermería. Docente Universidad Surcolombiana, Enfermera de la Unidad de Cuidados Intensivos del Hospital Universitario Hernando Moncaleano Perdomo de Neiva - Huila. E-mail: omairagt@gmail.com *** Enfermera, Especialista en Cuidado Intensivo, Magister en Educación. Docente Universidad de la Sabana. Bogotá. *** Enfermero, Magister en Epidemiología, aspirante a
\end{abstract} doctor en Estadística. Docente Universidad del Bosque. Bogotá - Colombia.}

Palabras clave: Cuidados de Enfermería; Prevención del delirium; Cuidados basados en evidencia; Modelo de Betty Neuman

Keywords: Delirium, Nursing Care; Prevention of delirium; evidence-based care; Betty Neuman Model

\section{RESUMEN}

Antecedentes: El Delirium es una alteración del estado de conciencia, frecuente en las unidades de cuidado intensivo. En la UCl adultos del Hospital Universitario de Neiva se tiene una incidencia del $28 \%$, esto aumenta la morbimortalidad; sin embargo es prevenible y requiere intervención.

Objetivo: Determinar la efectividad de los cuidados aplicados, según la Guía de enfermería elaborada con evidencia y en base al Modelo de Betty Neuman, para controlar estresores del entorno que pueden ocasionar Delirium, en pacientes hospitalizados en la unidad de cuidados intensivos adulto.

Metodología: Se realizó un estudio cuantitativo, transversal, pre-experimental, donde se aplicó la Guía de cuidados de enfermería a 49 pacientes. Se utilizó el diseño de preprueba y postprueba para conocer la percepción que tenían los pacientes de los estresores del entorno.

Resultados: Los cuidados aplicados según la Guía propuesta, fueron efectivos, ya que previnieron el delirium en el $94 \%$ de los pacientes, sólo tres presentaron el evento, representando una incidencia acumulada de 6.12 casos X 100 pacientes intervenidos (IC 95\%: $1.67-16.1$ ), evidenciado en la curva de Kaplan-Meier. La prueba de hipótesis de proporciones $(Z 0.05=-1.65)$ indica que la presencia de delirium es significativamente menor a la de 2011 (28\%). 
Conclusiones: Los cuidados aplicados según la Guía propuesta, lograron prevenir la aparición de delirium en $94 \%$ de los pacientes a pesar de sus antecedentes de factores de riesgo desencadenantes de delirium. De tal forma que, al minimizar la presencia de estresores del entorno, se favorece un ambiente creado agradable y familiar, y se constituye en un cuidado de enfermería fundamental para la prevención de delirium en UCl.

\section{ABSTRACT}

Background: Delirium is a fluctuating disturbance in consciousness that is highly prevalent in the intensive care unit. The adult ICU of the Hospital Universitario de Neiva shows an incidence of $28 \%$ of delirium patients, this increases morbidity and mortality rates among critical ones; nonetheless, this can be prevented if intervention occurs.

Objective: Determine the effectiveness of To determine the effectiveness of care applied, according to guide nursing care nursing care to prevent delirium in patients, who were hospitalized in the adult intensive care unit, the patients were exposed to environmental stressors.

Methodology: A quantitative, prospective and pre-experimental study was carried out to 49 patients who received assessment with the help of a nursing care guide. Besides the guide, a pretest and a posttest were carried out to evaluate the patients' perception on environmental stressors.

Results: Three out of the 49 patients experienced delirium; this represents a cumulative incidence of 6.12 delirium cases $\times 100$ patients $(\mathrm{Cl} 95 \%$ : 1.67-16.1), evident with the Kaplan-Merier curve. Based on the proportion hypothesis testing $\left(Z_{0.05}=-1.65\right)$ and in contrast to previous results where the incidence of delirium was of $28 \%$, the statistical evidence suffices to demonstrate that the presence of the syndrome is much less frequent in patients to whom the nursing care guide.

Conclusions: The guide prevented the occurrence of delirium in $94 \%$ of the patients, regardless of their deliriogenic history, by controlling the continuous noise and artificial light. This is why, minimizing environmental stressors, which results in a more pleasant environment, is a fundamental part of nursing care for the prevention of such a syndrome.

\section{INTRODUCCIÓN}

Los pacientes hospitalizados en la Unidad de Cuidado Intensivo (UCI) están expuestos a presentar o desarrollar Delirium o síndrome confusional, definido por la Americam Psychriatric Associations como una alteración de la conciencia, disminución de la capacidad de prestar atención al entorno, con reducción de la habilidad para centrar, mantener o dirigir la atención; se presenta con cambios en las funciones cognoscitivas como deterioro de la memoria, desorientación, alteración del lenguaje o presencia de una alteración perceptiva que no se explica por una demencia previa o en desarrollo. La alteración se presenta en un corto período de tiempo, habitualmente en horas o días y tiende a fluctuar a lo largo del día ${ }^{(1)}$.

La incidencia del delirium es alta en pacientes quirúrgicos, especialmente posterior a procedimientos cardiotorácicos, con una prevalencia del $32 \%{ }^{(2)}$; ortopédicos, como el post quirúrgico de fractura de cadera, con una incidencia del $35 \%{ }^{(3)}$. En población hospitalaria la mayoría de estudios reportan prevalencias entre el $10 \%$ y el $20 \%$, pero en $\mathrm{UCl}$, estudios que reportan unas tasas de más del $50 \%$ en grupos mixtos de pacientes médicos y quirúrgicos mayores de 60 años ${ }^{(4)}$. En la $\mathrm{UCl}$ adultos del Hospital Universitario de Neiva (HUN), la incidencia es del $28 \%$.

La fisiopatología del Delirium está determinada por un desequilibrio en la síntesis, liberación e inactivación de neurotransmisores que regulan el control de la función cognitiva, comportamiento y humor. La Dopamina, el ácido gama amino butírico 
(GABA) y la acetilcolina son los tres neurotransmisores involucrados en la fisiopatología del Delirium. La dopamina incrementa la actividad de las neuronas, mientras que el GABA y acetilcolina disminuyen la excitabilidad neuronal. El desequilibrio en estos neurotransmisores da como resultado inestabilidad neuronal y de la neurotransmisión ${ }^{(5,6)}$.

Existen factores de riesgo que favorecen que los pacientes hospitalizados en unidades de cuidado intensivo presenten delirium; dentro de estos factores están las anormalidades inflamatorias inducidas por endotoxinas y citosinas, como el factor de necrosis tumoral, hipoxemia, perfusión cerebral inadecuada, alteraciones metabólicas, terapia médica, como: ventilación mecánica y uso de medicamentos sedantes y analgésicos ${ }^{(7)}$. Jiménez et al clasifican los factores estresores para presentar Delirium según su etiología en factores propios del paciente, farmacológicos y externos o del ambiente ${ }^{(8)}$.

Del paciente: Edad sobre 70 años o más, procedencia de un asilo, Historia previa de depresión, demencia, de ictus, epilepsia, abuso de alcohol, sobredosis o uso de drogas ilegales, hipo o hipernatremia, hipo o hiperglicemia, hipo o hipertiroidismo, hipotermia o fiebre, Enfermedad renal, hepática, historia de falla cardíaca, shock séptico o cardiogénico, Infección por el VIH y Alimentación enteral, catéter vesical o renal, catéter venoso central, malnutrición.

Administración de fármacos: Psicoactivos, Sedantes-hipnóticos, Benzodiazepinas.

Externos: Ruido, Aislamiento físico y social, Luz artificial, Falta de reloj con calendario en unidades y Uso de restricciones físicas. (Inmovilizadores o medios de atadura).

Estas características propias de las Unidades de Cuidado Intensivo, como son el ruido constante de los equipos médicos, ventiladores mecánicos, bombas de infusión, monitores de signos vitales, grabadora, personal asistencial y celulares, junto con la iluminación artificial continua, hacen que la hospitalización en una UCl sea generadora de estrés y favorezca la alteración del sueño, junto con estrés evidenciado en desorientación, confusión e incluso psicosis en el paciente, factores desencadenantes de Delirium ${ }^{(9)}$.

De igual forma, diversos autores, confirman que la alteración del sueño, las inmovilizaciones y restricciones físicas, ocasionan déficit funcional y cognitivo, y son factores comunes en las $\mathrm{UCI}$, los cuales son estresantes que ocasionan Delirium ${ }^{(5),(9-}$ 11).

Entre los factores estresores presentes en este tipo de ambiente, Hewitt destaca la alteración sensorial, identificando algunos tipos de alteraciones ambientales que pueden conducir a un comportamiento anormal: como ruido que se hace fuerte y excesivo por el espacio cerrado de una UCI, ocasionado por bombas de infusión, monitores de signos vitales, ventiladores mecánicos, alarmas de estos equipos, teléfonos, voces y conversaciones del personal del equipo de salud, como no es posible valorar adecuadamente su nivel cognitivo y de conciencia, puede que lleguen a entender parcialmente o erróneamente esas conversaciones y les produzca miedo y ansiedad y, más aún, pueden alterar la cantidad y calidad de sueño ${ }^{(12)}$.

Pero es el ruido ambiental la principal causa en la interrupción del sueño en la UCI, cerca del $17 \%$ de los despertares frecuentes en los pacientes de UCI fueron 
ocasionados por ruido ${ }^{(13)}$. Estudios en $\mathrm{UCI}$ neonatal han comprobado que el ruido ha llegado a oscilar entre 64 y 71 decibeles, muy por encima de lo recomendado para las $\mathrm{UCl}$ que es de 45 decibeles en el día y 35 en la noche; las principales fuentes asociadas que identificaron fueron el tráfico exterior, factores humanos y equipos médicos ${ }^{(14-16)}$. Además, la luz artificial también incide en la alteración del sueño de los pacientes críticos, sin embargo, su control está en manos de la enfermera, por lo que debe ser adecuadamente administrada, ya que estudios reportan que la principal fuente de luz que incidía en el sueño de los pacientes era innecesaria ${ }^{(17)}$.

De este modo, esta alteración constituye un evento prevenible desde la identificación de los factores de riesgos existentes en un paciente hospitalizado en la unidad de cuidados intensivos, contando con un diagnóstico oportuno y la prestación de un cuidado de enfermería enfocado en la prevención y participación en el tratamiento del paciente ${ }^{(3)}$. Teniendo en cuenta que el cuidado de Enfermería debe estar fundamentado en conocimientos científicos del saber propio, es necesario realizar un planteamiento del cuidado desde los fundamentos teóricos que fortalecen esta profesión. Por tal motivo, para el presente estudio se busca aplicar el modelo de sistemas de Betty Neuman, el cual propone la prevención como intervención y plantea la identificación y el control de los factores estresores del entorno, para lograr retornar al paciente a su estado de bienestar ${ }^{(18)}$, por lo que es factible y de gran utilidad para la prevención complicaciones del paciente hospitalizado en cuidado intensivo, como lo es el Delirium.

Así, prevenir el delirium en $\mathrm{UCl}$ reduce riesgos para el paciente, disminuye su estancia en UCI, días de ventilación mecánica, riesgos de eventos adversos, disminuye costos emocionales y económicos para el paciente, familia y sistema de salud $^{(15)}$.

El objetivo general del presente estudio fue: Determinar la efectividad de los cuidados aplicados, según la Guía de enfermería elaborada en base al Modelo de Betty Neuman y con Enfermería Basada en Evidencia (EBE), para controlar estresores del entorno, ruido y luz artificial de día y noche, con su consecuente alteración del sueño, factores que pueden ocasionar Delirium, en pacientes hospitalizados en la Unidad de Cuidados Intensivos Adulto (UCl-A) del Hospital Universitario de Neiva (HUN).

Se platearon dos Hipótesis:

Ho: La proporción del Delirium en pacientes intervenidos con la guía de cuidados de enfermería basada el modelo de Betty Neuman y en EBE es igual a la proporción de Delirium existente UCl adultos polivalente del HUN.

Ha: La proporción del Delirium en pacientes intervenidos con la guía de cuidados de enfermería basada el modelo de Betty Neuman y en EBE es menor a la proporción de Delirium existente UCl adultos polivalente del HUN.

\section{METODOLOGÍA}

Estudio cuantitativo, prospectivo pre-experimental. Siguiendo el diseño de preprueba y postprueba, se contó con un único grupo de pacientes, al que se le aplicó la guía de cuidados de enfermería para prevenir el delirium y se indicó la proporción de delirium en estos pacientes. Se tomó un tipo de muestreo probabilístico aleatorio simple. Para el cálculo del tamaño de la muestra, se utilizó la fórmula específica para estudios 
experimentales, descrita por Desu y Raghavarao ${ }^{(19)}$. Así, el tamaño de muestra adoptado fue de 49 pacientes, asumiendo $\alpha=0.05, \beta=0.2$ y $\delta^{*} \min =0.25268026$.

\section{Instrumentos}

Se elaboró la preprueba, postprueba y la guía de cuidados de enfermería según los conceptos del modelo de Betty Neuman, dando así lugar a la aplicación de su teoría de sistemas, ésto a través de la prevención secundaria para el fortalecimiento de las líneas de resistencias y la reconstitución en el paciente en UCI.

La Guía de cuidados de enfermería para controlar estresores del entorno que pueden ocasionar delirium se elabora partiendo del modelo de Betty Neuman, teniendo en cuenta los factores estresores del entorno, ruido y luz artificial de día y de noche, con su consecuente alteración del sueño, factores desencadenantes del delirium; además se empleó la evidencia científica con algunos lineamientos del protocolo desarrollado por Inouye et al para el desarrollo de la investigación A multicomponent intervention to prevent Delirium in hospitalized older patients ${ }^{(20)}$, otros descritos por los enfermeros Rodríguez et al en su investigación Síndrome confusional agudo en $\mathrm{UCl}$, factores que influyen y actitud de enfermería ${ }^{(21)}$ y la Guía para el diagnóstico y tratamiento del Hospital Universitario Central de Asturias, dispuesta por Ruiz et al (22); junto con la clasificación planteada por Alberto Galvez Toro, quien adaptó la clasificación utilizada en Medicina Basada en la Evidencia a Enfermería basada en la Evidencia (EBE) incluyendo la investigación cualitativa ${ }^{(23)}$, además se tiene en cuenta la clasificación de la evidencia, atendiendo a la utilidad, relacionada con la resolución o no del problema $^{(24)}$.

La Guía, está orientada a reduclr los estresores del entorno ruido y luz artificial continua, con su consecuente alteración del sueño, de forma que busca que los pacientes se sientan en un ambiente creado familiar y agradable, que les permita dormir (Ver Tabla I).

Tabla I: Guía de cuidados de enfermería para controlar estresores del entorno posibles desencadenantes de delirium en $\mathrm{UCl}$, basada en la teoría de Betty Neuman y en enfermería basada en la evidencia

\begin{tabular}{|c|c|c|c|}
\hline CUIDADO DE ENFERMERÍA & META & JUSTIFICACIÓN & EBE \\
\hline Saludar al paciente por su nombre (25). & \multirow{3}{*}{$\begin{array}{l}\text { Favorecer la interacción } \\
\text { y promover el bienestar } \\
\text { en el paciente al } \\
\text { mantenerlo informado de } \\
\text { los cambios en el } \\
\text { entorno, relacionados } \\
\text { con el ruido y la luz } \\
\text { artificial continua y } \\
\text { demás intervenciones. }\end{array}$} & \multirow{3}{*}{$\begin{array}{l}\text { "Se relacionan mayores niveles de } \\
\text { malestar emocional en los pacientes } \\
\text { que indican tener peor comunicación } \\
\text { (No comunicar a su cuidador su } \\
\text { preocupación, no mantener } \\
\text { comunicación con el cuidador) }{ }^{(25)} \text {. }\end{array}$} & \multirow{3}{*}{$\begin{array}{l}\text { Nivel de } \\
\text { Evidencia } 4 . \\
\text { Nivel de } \\
\text { Utilidad Alfa }\end{array}$} \\
\hline $\begin{array}{l}\text { Indicar al paciente las características de } \\
\text { la unidad, horarios de visita, } \\
\text { alimentación, baño y demás aspectos } \\
\text { administrativos de la unidad (25). }\end{array}$ & & & \\
\hline $\begin{array}{l}\text { Informar al paciente todas las actividades } \\
\text { y procedimientos que se le van a realizar, } \\
\text { como procedimientos, curaciones, } \\
\text { administración de medicamentos, } \\
\text { cambios de posición y valoración } \\
\text { física }^{(20) .}\end{array}$ & & & \\
\hline
\end{tabular}




\begin{tabular}{|c|c|c|c|}
\hline CUIDADO DE ENFERMERÍA & \multirow[t]{2}{*}{ META } & \multirow[t]{2}{*}{ JUSTIFICACIÓN } & \multirow[t]{2}{*}{ EBE } \\
\hline $\begin{array}{l}\text { Explicar al paciente posibles ruidos que } \\
\text { puede llegar a escuchar, producidos por } \\
\text { bombas de infusión, alarma del monitor, } \\
\text { del ventilador y demás }{ }^{(20)}\end{array}$ & & & \\
\hline $\begin{array}{l}\text { Dialogar frecuentemente con el paciente, } \\
\text { proporcionándole información acerca de } \\
\text { datos de ubicación espacial y temporal, } \\
\text { información sobre su estado de salud y } \\
\text { pronóstico si es posible, tratamientos que } \\
\text { se están administrando, situación familiar } \\
\text { y dar respuesta a las demás inquietudes } \\
\text { que exprese } \\
{ }^{(20)} \text {. }\end{array}$ & $\begin{array}{l}\text { Favorecer patrones de } \\
\text { sueño durante el tiempo } \\
\text { que el paciente } \\
\text { permanezca en la UCl, } \\
\text { evitando el sueño diurno } \\
\text { y reducir la actividad en } \\
\text { el horario nocturno }\end{array}$ & $\begin{array}{l}\text { Las estrategias no farmacológicas } \\
\text { incluyen la reorientación, la } \\
\text { estimulación cognitiva varias veces al } \\
\text { día, adecuar la relación sueño-vigilia, } \\
\text { la estimulación visual y auditiva, y } \\
\text { minimizar en lo posible el ruido y la } \\
\text { luz artificial. Con estas intervenciones } \\
\text { se reduce hasta en un } 40 \% \text { la } \\
\text { incidencia del delirio. Deberá } \\
\text { informarse a los familiares sobre } \\
\text { estas alternativas de manejo y } \\
\text { explicárselas para que no les cause } \\
\text { ansiedad }{ }^{(27)} \text {. }\end{array}$ & $\begin{array}{l}\text { Grado de } \\
\text { Recomenda- } \\
\text { ción Fuerte. } \\
\text { Nivel de } \\
\text { Evidencia 1B }\end{array}$ \\
\hline $\begin{array}{l}\text { Conversar con el paciente } \\
\text { ratificándole su condición y su } \\
\text { orientación en tiempo y lugar }{ }^{(28)}\end{array}$ & $\begin{array}{l}\text { Proporcionar un } \\
\text { ambiente de comodidad } \\
\text { y descanso al paciente } \\
\text { durante el tiempo que } \\
\text { permanezca en la UCl }\end{array}$ & $\begin{array}{l}\text { "Orientar al paciente con los } \\
\text { nombres de los miembros del equipo } \\
\text { de atención y día calendario y la } \\
\text { comunicación para reorientar en los } \\
\text { alrededores, son actividades } \\
\text { cognitivamente estimulantes que } \\
\text { mejoran significativamente } \\
\text { la orientación y muestra una } \\
\text { significativa } \\
\text { reducción en la tasa de uso de } \\
\text { sedantes para } \\
\text { dormir" (20) }\end{array}$ & $\begin{array}{l}\text { Nivel de } \\
\text { Evidencia } 2 \text { - } \\
\text { Utilidad Alfa }\end{array}$ \\
\hline $\begin{array}{l}\text { Estimular los estados de ALERTA en el } \\
\text { paciente (favoreciendo la lectura) } \\
\text { permitiendo el ingreso de elementos } \\
\text { personales como anteojos, revistas, } \\
\text { libros de interés personal, agenda y } \\
\text { libros personales; para que el paciente } \\
\text { mantenga el ciclo circadiano, ayudando a } \\
\text { que escuche música, lea, vea revistas, } \\
\text { periódico, mantenga conversaciones con } \\
\text { la familia o personal de salud durante el } \\
\text { día y en la noche duerma. Para esto se } \\
\text { permitirá el ingreso de elementos } \\
\text { personales como anteojos, revistas, }\end{array}$ & & $\begin{array}{l}\text { "Orientar al paciente con los } \\
\text { nombres de los miembros del equipo } \\
\text { de atención y día calendario y la } \\
\text { comunicación para reorientar en los } \\
\text { alrededores, son actividades } \\
\text { cognitivamente estimulantes que } \\
\text { mejoran significativamente } \\
\text { la orientación y muestra una } \\
\text { significativa } \\
\text { reducción en la tasa de uso de } \\
\text { sedantes para } \\
\text { dormir" }{ }^{20} \text { ) }\end{array}$ & $\begin{array}{l}\text { Nivel de } \\
\text { Evidencia } 6 . \\
\text { Utilidad Alfa }\end{array}$ \\
\hline grabadora, agenda y libros personales ${ }^{(20)}$. & & "Los efectos del ruido por encima de & \\
\hline
\end{tabular}




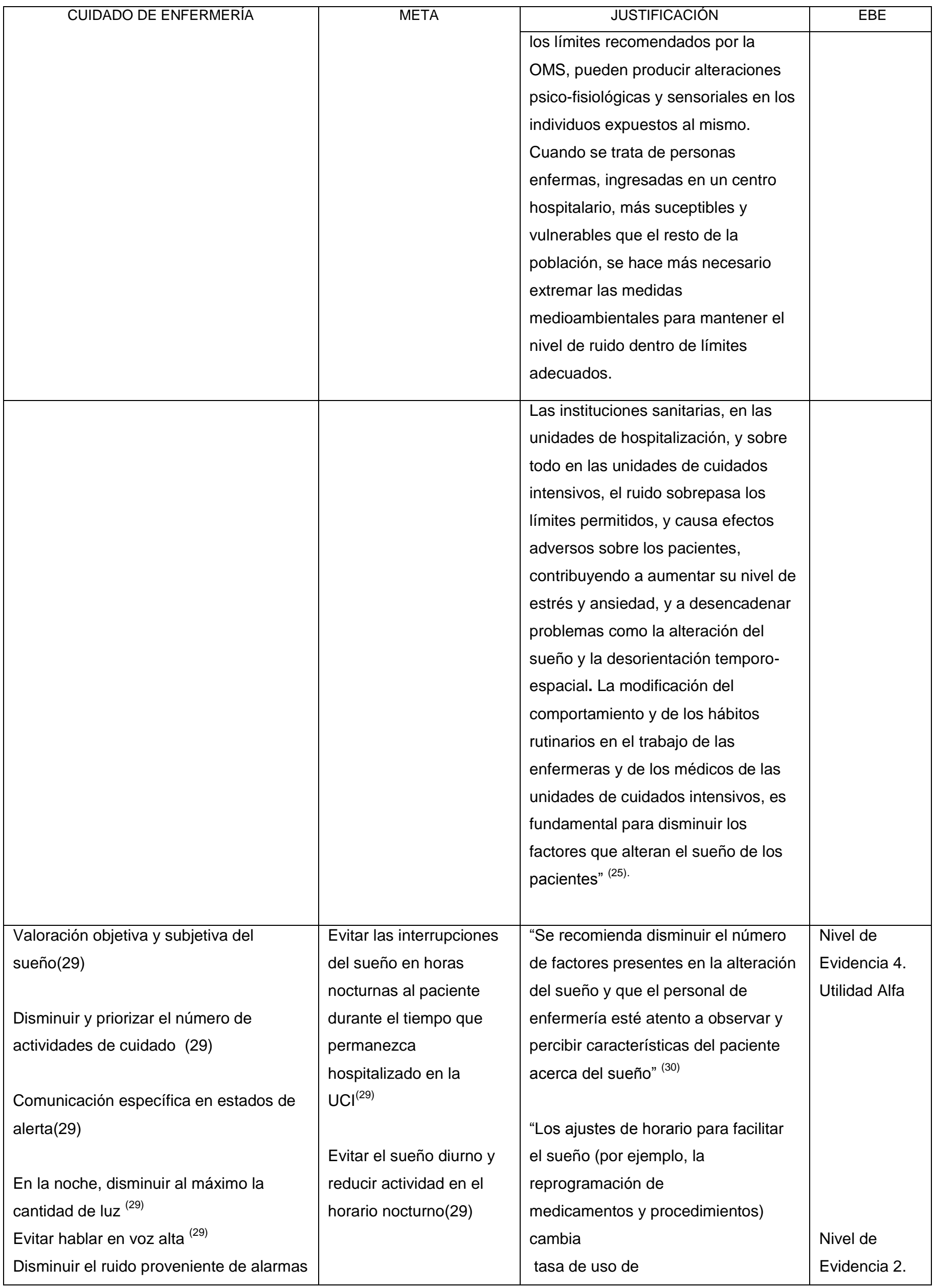




\begin{tabular}{|c|c|c|c|}
\hline CUIDADO DE ENFERMERÍA & META & JUSTIFICACIÓN & EBE \\
\hline $\begin{array}{l}\text { de monitores y ventiladores }{ }^{(29)} \\
\text { Disminuir el volumen de los timbres } \\
\text { telefónicos y evitar tareas ruidosas }{ }^{(29)} \\
\text { No interrumpir los periodos de sueño, } \\
\text { evitando administrar tratamientos, } \\
\text { actividades de enfermería o médicas, } \\
\text { sin empeorar la condición del paciente }{ }^{(29)} \\
\text { Responder rápidamente a apagar las } \\
\text { alarmas }{ }^{(31)} \\
\text { Apagar radios en la unidad } \\
\text { Situar a los pacientes más lábiles lejos } \\
\text { de las áreas de alto tráfico }{ }^{(31)}\end{array}$ & & medicamento sedante para dormir"(20) & Utilidad Alfa \\
\hline
\end{tabular}

\section{MÉTODO Y TÉCNICA}

Se inició con el reclutamiento de los pacientes que cumplían con los criterios de selección, los cuales incluyen criterios de inclusión y exclusión, siendo los de inclusión:

$\checkmark$ Encontrarse en una escala RASS entre -3 y +3

$\checkmark$ Firma de consentimiento

$\checkmark$ No tener Delirium (CAM-ICU Negativo)

$\checkmark$ Edad mayor de 23 años

Y de exclusión:

$\checkmark$ Padecer algún trastorno que neurológico o psiquiátrico que le impida mantener estado de conciencia.

Posterior a este proceso, se recolectó la información pertinente a la línea de base, se aplicó la preprueba para conocer la percepción del paciente frente a los estresores del entorno. Se continuó con la aplicación de la guía de cuidados de enfermería, lo cual se hizo de forma personalizada, según las necesidades y características de cada paciente; ésta fue aplicada, junto con las escalas RASS y CAM-ICU, de forma continua, en cada turno, mañana, tarde y noche, mínimo por cinco días. Teniendo en cuenta estos criterios el tamaño total de la muestra es de 49 pacientes.

Finalmente, al quinto día, luego de aplicada la guía de cuidados continuamente por los cinco días, se realizó la postprueba, test que contiene los mismos puntos evaluados en la preprueba, donde se valoró la respuesta de los participantes frente a las variables intervenidas con la guía de cuidados. La información recolectada fue procesada y analizada a través del software Epilnfo 3.5.3 y STATA (Versión 10 SE; Stata Corporation, College Station, Texas). A través del software Stata se obtuvo herramientas estadísticas como la curva de supervivencia de Kaplan Meier para el análisis de la aparición de delirium en los participantes; análisis de proporciones estadísticas y prueba Shapiro - Wilk para la variable edad.

\section{RESULTADOS}

Se inició el estudio con la recolección de la línea de base, es decir la concreción de 
las características de los pacientes, tanto fisiológicas como biológicas y de percepción del entorno que tenían los pacientes del estudio, encontrándose que el $77,5 \%$ de los participantes fueron hombres; la edad promedio fue de 50 años y éstas variaron entre 23 años el más joven y 92 años el de mayor edad. Acerca de los factores de riesgo para el delirium, el $71.4 \%$ de los participantes al momento del reclutamiento tenían dispositivos médicos, como catéter venoso central, sonda vesical, sonda gástrica para alimentación o drenaje, tubos de drenaje tipo mediastinal.

Las enfermedades infecciosas y las cirugías cardiotorácicas fueron los principales antecedentes de los participantes ( $22.4 \%$ cada uno) y los diagnósticos más frecuentes fueron el de postoperatorio de cirugía cardiotorácica tipo Revascularización Miocárdica o cambio valvular y el de síndrome coronario agudo tipo infarto agudo de miocardio (20.4\% cada uno). Acerca de la escala RASS, al ingreso al estudio, los participantes se encontraban en su gran mayoría (67\%), en RASS 0, es decir alerta y tranquilos. De las variables biológicas, niveles de sodio, glucemia y temperatura se mantuvieron en rangos normales en todos los participantes durante los cinco días de seguimiento. Sobre los factores farmacológicos, el $3 \%$ de los participantes tuvo sedantes, el $56 \%$ analgésicos (de tipo fentanyl y morfina) y el $8 \%$ vasoactivos.

De los 49 pacientes a quienes se les aplicó la guía, tres presentaron delirium, representando una incidencia acumulada de 6.12 casos de delirium $X 100$ pacientes intervenidos y una densidad de incidencia de 5.19 casos $X 10.000$ pacientes-día (IC 95\%: 1.67 - 16.1), como se evidencia en la tabla II. La probabilidad de no presentar delirium dado que se estaba bajo la aplicación de la guía de cuidados de enfermería, al inicio del estudio era del 97.9\%, cifra que disminuyó hasta el $93.8 \%$ al día cinco de aplicación de la guía de cuidados.

Tabla II. Funciones de supervivencia para los eventos presentados durante el seguimiento

\begin{tabular}{cccccc}
\hline \multirow{2}{*}{$\begin{array}{c}\text { Tiempo } \\
\text { (horas) }\end{array}$} & $\mathrm{Nj}$ & $\mathrm{Dj}$ & $\mathrm{C}$ & $\mathrm{F}^{*}$ & $\mathrm{IC} \mathrm{95 \%}$ \\
\cline { 2 - 6 } & 49 & 0 & 0 & 1 & 1 \\
\hline 0 & 49 & 1 & 0 & 0.9796 & $0.86-0.99$ \\
\hline 81,5 & 48 & 1 & 0 & 0.9592 & $0.84-0.98$ \\
\hline 109,5 & 47 & 1 & 0 & 0.9388 & $0.82-0.97$ \\
\hline 120 & 46 & 0 & 0 & 0.9388 & $0.82-0.97$ \\
\hline Tiempo = Tiempo en horas hasta que se presenta el evento (delirium) & \\
\hline $\mathrm{Nj}=$ Número de pacientes supervivientes & \\
\hline Dj = Número de eventos en el tiempo & \\
\hline $\mathrm{C}=$ Censura (Datos perdidos o censurados) \\
\hline $\mathrm{F}=$ Función de supervivencia calculada por el método de Kaplan - Meier. \\
\hline $\mathrm{IC}=$ Índice de confianza \\
\hline
\end{tabular}

La tabla II, describe la función de supervivencia en tiempo, entendiendo supervivencia a la no presencia de delirium, por lo que la tabla describe el tiempo que tardaron los tres pacientes en presentar el evento. 
De manera general, aunque se presentaron casos de delirium bajo la aplicación de la guía de cuidados, se evitó la aparición del evento en el $94 \%$ de los pacientes. Este comportamiento del riesgo se evidencia en el gráfico de curva de supervivencia de Kaplan - Meier (figura 1).

Figura 1. Curva de supervivencia de los pacientes tratados bajo la aplicación de la guía de cuidados de enfermería.

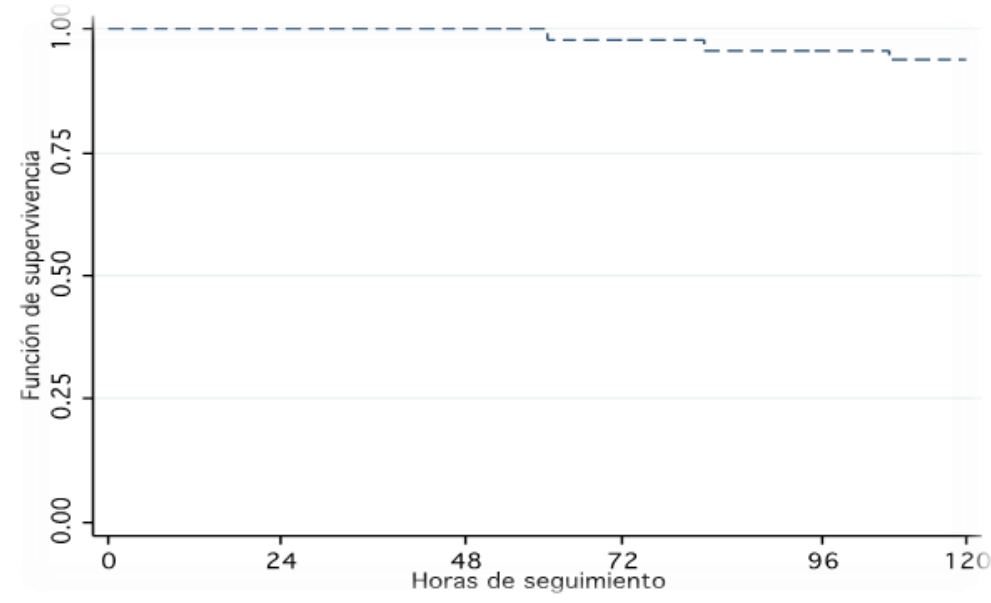

En la curva de supervivencia de Kaplan Meier se observa el tiempo que tardaron los pacientes en presentar el evento, debido a la poca presencia de delirium, se observa la poca disminución de la curva, por lo que la guía de cuidados de enfermería previno el delirium.

Además de esto, teniendo en cuenta las hipótesis nula y alterna planteadas en el presente estudio, se realiza una prueba de hipótesis de proporciones, con resultado de $Z_{0.05}=-1.65$, lo que demuestra suficiente evidencia estadística para establecer que la presencia de delirium es mucho menor en los 49 pacientes en quienes se aplicó la guía de cuidados de enfermería, frente al porcentaje previo de delirium, correspondiente al $28 \%$.

Acerca de los estresores del entorno, en la preprueba $89.7 \%$ de los pacientes tenían problemas para conciliar el sueño, pero luego de aplicar la guía de cuidados, hubo una gran reducción al $24.48 \%$, de forma que la guía mejoró la cantidad de sueño en los pacientes intervenidos, sólo 12 pacientes continuaban con dificultades para conciliar el sueño, de ellos dos pacientes fueron los que presentaron delirium.

Se encontró que el ruido es el principal estresor desencadenante de alteración del sueño, según refieren los participantes en la preprueba, sin embargo, se observa una reducción significativa de éste y de la luz artificial, en la postprueba, luego de la aplicación de los cuidados que modificaban el entorno de la UCI, a través del control de estresores ruido y luz artificial continua, de tal forma que reduciendo el ruido y la luz artificial los pacientes lograron dormir y no presentaron delirium. En la figura 2 se evidencia una reducción significativa de la percepción del ruido y luz artificial continua, de los participantes en la postprueba, como estresores que no permiten dormir. 
Figura 2. Percepción acerca de las causas que ocasionan dificultad para conciliar el sueño.

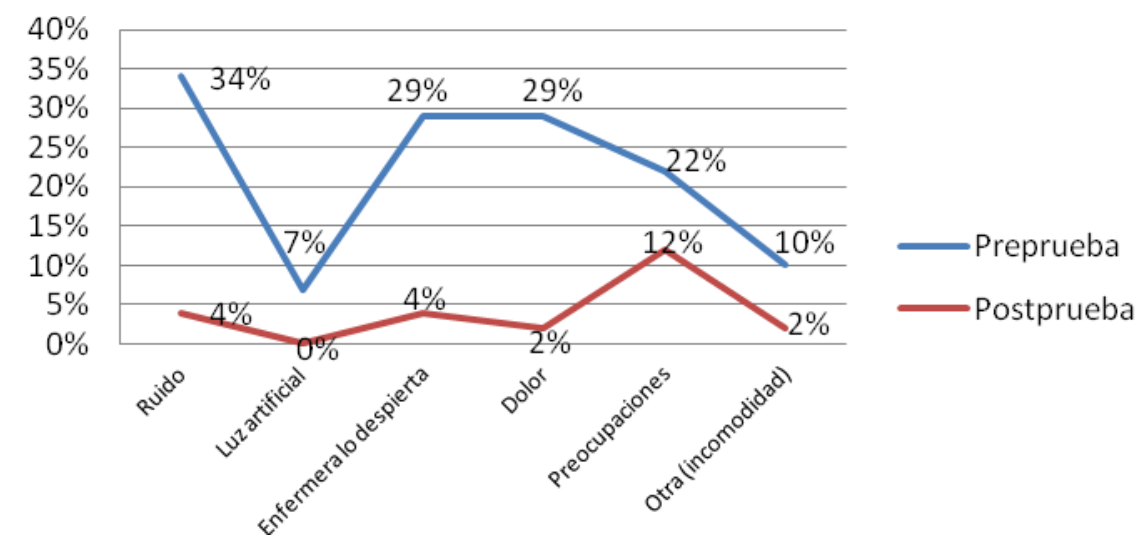

La figura 2 evidencia el cambio significativo que tuvieron los pacientes en su percepción del entorno, correspondiente al ambiente de la $\mathrm{UCl}$, el cual mejoró posterior a la aplicación de la guía de cuidados de enfermería, favoreciendo en los pacientes un mejor entorno que le propició sueño y evitó el delirium.

\section{DISCUSIÓN}

A través del análisis de la información obtenida se responde a la pregunta de investigación y se comprueba la hipótesis alterna planteada, al evidenciar que la guía de cuidados de enfermería para prevenir el delirium en $\mathrm{UCl}$, sí fue efectiva, a pesar de la presencia de factores de riesgo para el delirium de tipo farmacológicos y propios del paciente.

Los factores de riesgo para el delirium, como alteraciones del sodio, de la glucemia, enfermedad infecciosa, enfermedad grave, hipertermia y la administración de sedantes como midazolam, analgésicos como morfina y fentanyl, dispositivos médicos, antecedentes de cáncer, de cirugía cardiotorácica y ortopédica, se tuvieron en cuenta en la recolección de la información y se plantearon en la línea de base de cada paciente, se confirma lo que ha sido ampliamente descritos por Inouye (5), Wesley ${ }^{(10)}$, entre otros, como causales de delirium; por lo tanto, la presencia de estos factores en los pacientes los hace más vulnerables para presentar el evento; comprobado en este estudio, debido a que los tres pacientes que presentaron delirium, tenían presentes estos factores. Sin embargo, con la guía de cuidados de enfermería, fue posible prevenir el delirium en pacientes con estos factores de riesgo; ya que fue posible controlar factores del ambiente que reducían la posibilidad de sueño en el paciente.

De este modo, en la presente investigación, la reducción de los estresores ruido y luz artificial continua, con su consecuente alteración del sueño, logró mejorar el sueño en los pacientes, ya que un $89 \%$ refirió tener dificultades para dormir en la preprueba; pero luego de la aplicación de la guía de cuidados, este porcentaje se redujo a un $24 \%$, es decir que minimizar las fuentes de ruido y luz artificial continua, favorecen el sueño en los pacientes hospitalizados en $\mathrm{UCl}$, manifestado por los pacientes y evidenciado en sus amplios periodos de sueño y en sus actitudes de ánimo, colaboración y tranquilidad. Así, se redujo el estrés y las posibilidades de padecer delirium; esto descrito por Blanco et al, junto con otros autores, quienes confirman que la alteración del sueño, ocasionan déficit funcional y cognitivo, y son factores 
estresantes que causan Delirium ${ }^{(9,10,20)}$.

Los tres pacientes que presentaron delirium tenían antecedentes influyentes en su aparición, como múltiples dispositivos médicos de tipo catéter venoso central, línea arterial y sonda vesical, para su monitoría hemodinámica y la administración del tratamiento farmacológico; esto es confirmado por Jiménez ${ }^{(8)}$, quien lo clasifica como estresor propio del paciente, que favorece la presencia de delirium, de forma que la presencia de múltiples factores aumenta la posibilidad de padecer delirium.

Los resultados encontrados evidencian que es posible prevenir el delirium en UCI, a través del control de estresores del entorno, es decir, garantizando al paciente periodos prolongados de sueño; sin embargo, a pesar de que se aplicó la guía de cuidados de enfermería de forma continua, en cada turno durante los cinco días dispuestos, no fue posible evaluar durante más días la ocurrencia del evento en pacientes de estancia prolongada, para confirmar la efectividad de la guía y además no se controlaron otros factores que favorecen el delirium, como los propios del paciente y los farmacológicos, lo cual fue una limitante para el presente estudio; sin embargo, como describe el modelo de Betty Neuman, mantener un ambiente creado agradable reduce las probabilidades de afectar las líneas de resistencia y mantener una prevención secundaria, como fue en el presente estudio, evitar o prevenir el delirium.

\section{CONCLUSIONES}

Los cuidados de enfermería aplicados según la Guía elaborada basada en la teoría de Betty Neuman y en enfermería basada en evidencia, logró prevenir la aparición de delirium en $94 \%$ de los pacientes a pesar de sus antecedentes de factores de riesgo. De tal forma que, al minimizar la presencia de estresores del entorno, se favorece un ambiente creado agradable y familiar, y se constituye en un cuidado de enfermería fundamental para la prevención de delirium en UCI.

Es posible aplicar la teoría de enfermería, en este caso la de Betty Neuman, como fundamento del cuidado de Enfermería, de manera que hace un gran aporte a la disciplina al estructurar cuidados que resuelven las necesidades de los pacientes.

\section{Recomendaciones}

Es conveniente aplicar la guía de cuidados de enfermería para prevenir el delirium en UCI, teniendo en cuenta las necesidades de los pacientes, es decir personalizar los cuidados de la guía, de forma que se responda a dichas necesidades, como se realizó en este estudio a través del manejo del dolor, de las preocupaciones, del estrés, de la incomodidad. Debe continuarse realizando investigaciones donde enfermería haga sus aportes con más cuidados que controlen otras variables deliriogénicas como reducción de medicamentos opioides, benzodiacepinas, control de sodio, glucemia y temperatura.

\section{REFERENCIAS}

(1) APA. American Psychiatric Association. [Internet] Washington Diagnostic and statistical manual of mental disorders, 4th ed. Washington DC: 1994. [citado marzo 2011] Disponible en: http://psycnet.apa.org/psycinfo/1994-97860-000 
(2) Rincon Hg, Granados M, Unutzer J, Gomez M, Duran R, Badiel M et al. Prevalence, detection and treatment of anxiety, depression, and delirium in the adult critical care unit. Psychosomatics [Internet]. 2001; [citado abril 2011]; 42(5):391-396. Disponible en: http://www.sciencedirect.com/science/article/pii/S0033318201704707

(3) Litaker D, Locala J, Franco K, Bronson DI, Tannous Z. Preoperative risk factors for postoperative delirium. Gen Hosp Psychiatry [Internet]. 2001; [citado abril 2011];23(2):84-89. Disponible en: http://www.ncbi.nlm.nih.gov/pubmed/11313076

(4) Florez P. Velasquez J. Frecuencia y factores de riesgo del delirium en población geriátrica de la unidad de cuidados intensivos de la clínica Reina Sofía en Bogotá. Revista médica Sanitas [Internet]. 2009. [citado Mayo 2012];12(3):66-73. Disponible en: http://www.unisanitas.edu.co/Revista/9/quinto.pdf

(5) Inouye S. Delirium in Older Persons. Review article. The New England of Medicine. Massachusetts [Internet]. 2006; [citado agosto 2011]; 354:1157-1165. Disponible en: http://www.jewishhospitalcincinnati.com/files/Delirium in Older Persons.pdf

(6) Martinez G. Delirium respuestas pendientes. Revista Hospital clínico Universidad de Chile. [Internet]. 2008 [citado marzo 2012];19:330-8. Disponible en: http://www.redclinica.cl/HospitalClinicoWebNeo/Controls/Neochannels/Neo CH6258/d eploy/delirium.pdf

(7) Caceres A. Alva J. Manual de psiquiatría. Trastornos mentales orgánicos [Internet]. 2 ${ }^{\mathrm{a}}$ Ed capítulo 10. Lima: UNMSM, 1998 [Actualizado 2002-2008; citado marzo 2011] Disponible en:

www.sisbib.unmsm.edu.pe/bibvirtual/libros/psicologia/manual psiquiatría/cap-10.htm

(8) Jimenez $M$, Santeadoro $A$, Lanchado $E$. Delirium o síndrome confusional agudo. Tratado de geriatría para residentes. Sociedad española de geriatría y gerontología. [Internet] pdf. [citado marzo 2012]; Capítulo.18.Pag189. Disponible en: http://www.segg.es/download.asp?file=/tratadogeriatria/PDF/S35-05\%2018 II.pdf.

(9) Ayllon N. Garrido M. Álvarez M. Gonzalez G. Incidenciais del delirium en cuidados intensivos y factores relacionados. Enferm intensiva, [Internet]. 2007; [citado junio. 2011]; 18(3):138-43. Disponible en: http://zl.elsevier.es/es/revista/enfermeriaintensiva-142/incidencia-delirio-cuidados-intensivos-factores-relacionados-13109377originales-2007.

(10) Wesly E., Ayumi S., Truman B., Speroff Th., Gordon S, Delirium as a Predictor of Mortality in Mechanically Ventilated Patients in the Intensive Care Unit. United States. JAMA, [Internet]. 2004; [citado mayo 2011];291(14):1753-1762. Disponible en: http://www.ncbi.nlm.nih.gov/pubmed/15082703

(11) Raul Carrillo E, Sosa García J. Delirium en el enfermo grave. Medicina interna México. [Internet] 2010; [citado zomar 2012];26(2):130-139. Disponible en: http://www.slideshare.net/sandrakitty/48077693-deliriumenelenfermograve1

(12) Hewitt J. Psyco-affective disorder in intensive care units: a review. Journal of clinical Nursing [Internet] 2002, [citado abril 2012];11,(5):575-584. Disponible en: http://www.ncbi.nlm.nih.gov/pubmed/12201884

(13) Freedman N, Gazendam J, Levan L, Pack A, Schwab R. Abnormal Sleep/Wake Cycles and the Effect of Environmental Noise on Sleep Disruption in the Intensive Care Unit. American Journal of respiratory and critical care medicine. [Intertet]. 2001. [citado sep. 2011];163(2):451-457. Disponible en: http://www.ncbi.nlm.nih.gov/pubmed/11179121

(14) Centeno D, Apac A, Sánchez J, Raffo M, Centeno C. Niveles de ruido y fuentes asociadas en una Unidad de Cuidados Intensivos Neonatal. Revista Peruana de Pediatría [Intertet]. 2005. [citado Mayo 2012];58.(1):12-14. Disponible en: http://sisbib.unmsm.edu.pe/bvrevistas/rpp/v58n1/pdf/a03.pdf 
(15) Lawson N, Thompson K, Saunders G, Saiz J, Richardson J, Brown J, Ince N, Caldwell M, Papa D. Sound intensity and noise evaluation in a critical care unit. American association of critical - care nurses [Intertet]. 2010. [citado Sep. 2011];19.(6):88-98. Disponible en: http://www.ncbi.nlm.nih.gov/pubmed/21041190

(16) Fajardo D, Gallejo S, Argote L. Niveles de ruido en la Unidad de Cuidados Intensivos Neonatal "CIRENA" del Hospital Universitario del Valle, Cali - Colombia. Colombia Médica [Internet]. 2007. [citado Ene. 2012];38(4). Disponible en:

\section{http://www.bioline.org.br/pdf?rc07077}

(17) Dunn H, Anderson M, Hill P. Nighttime lighting in intensive care units. American association of critical - care nurses [Internet]. 2010. [citado Sep. 2011];30.(3): 31-37. Disponible en: http://ccn.aacnjournals.org/content/30/3/31

(18) Neuman B. Fawcett J. The Neuman Systems Model. Vol 1. $4^{\text {th }}$ edition. BostonMassachusetts: Prentice Hall; 2002.

(19) M.M. Desu, D. Raghavarao. Sample Size Methodology. Statistical Modeling and Decision Science. Vol 1.New York: Academic Press, Inc.1997

(20) Inouye S, Bogardus S, Charpentier P, Summers L, Acampora D, Holford T, Cooney L. A Multicomponent Intervention to Prevent Delirium in Hospitalized Older Patients. The New England Journal of medicine. Massachusetts medical society [Internet]. 1999; [citado marzo 2011]; 340(9):669-676. Disponible en: http://www.nejm.org/doi/full/10.1056/NEJM199903043400901\#t=article

(21) J. J. Rodriguez Mondéjar, R. Martín Lozano, R. M. Jorge Guillem, J. Iniesta Sánchez, C. Palazón Sánchez, F. Carrión Tortosa, F. Parra Dormal, A. López Amorós, M. Martínez Huéscar. Síndrome confusional agudo en $\mathrm{UCl}$ : factores que influyen y actitud de enfermería. Enferm intensiva, [Internet]. 2001; [citado abril 2011];12(1):3-9. Disponible en: http://zl.elsevier.es/es/revista/enfermeria-intensiva-142/sindromeconfusional-agudo-uci-factores-que-influyen-12003852-articulos-originales-2001

(22) Ruiz M, Mateos V, Suarez H, Villaverde P. Síndrome Confusional Agudo (Delirium), Diagnóstico y tratamiento. Hospital universitario Central de Asturias Oviedo. [internet] 2010. [citado mayo 2012] pdf, Pag 10. Disponible en: http://www.hca.es/huca/web/contenidos/websdepartam/pqe/GUIA\%20SINDROME\%2 OCONFUSIONAL\%20AGUDO.pdf

(23) Galvez Toro Alberto. Un enfoque crítico para la construcción de la enfermería basada en la evidencia. Rev. Investigación y educación en enfermería. [Internet] 2003, [citado agosto 2012];21(1):50-63. Disponible en: http://aprendeenlinea.udea.edu.co/revistas/index.php/iee/article/viewFile/3037/2805

(24) Orellana A. y Paravic T. Enfermería basada en evidencia. Barreras y estrategias para su implementación. Revista Ciencia y Enfermería de Chile. [Internet] 2007, [citado agosto 2012]; 13 (1). Disponible en: http://www.scielo.cl/scielo.php?script=sci_arttext\&pid=S0717-95532007000100003

(25) Perea Baena Mc. El silencio en la UCI ¿Una utopía? Evidentia. [Internet] 2006 [citado abril 2012]; 3(10). Disponible en: http://www.indexf.com/evidentia/n10/241articulo.php [ISSN: 1697-638X].

(26) Archury D y Archury L. Sueño en el paciente crítico: una necesidad insatisfecha en la Unidad de Cuidados Intensivos. Investigación en Enfermería: Imagen y desarrollo [Internet] 2010 [citado abril 2012]; Vol. 12 №1. Disponible en: http://www.redalyc.org/pdf/1452/145216903003.pdf.

(27) Celis E, Besso J, Birchenallc, De La Cal M, Carrillo R, Castorena G, Ceraso D, Dueñas C, Gil F, Jiménez E, Meza J, Muñoz M, Pacheco C, Palizas F, Pinilla D, Raffan F, Raimondi N, Rubiano S, Suarez M y Ugarte S. Guía de práctica clínica basada en la evidencia para el manejo de la sedo-analgesia en el paciente adulto críticamente enfermo. Medicina intensiva Madrid, [Internet] 2007 [citado abril 2012]; 
http://www.fsfb.org.co/sites/default/files/guiasdesedacionyanalgesia.pdf

(28) DUEÑAS C, ORTIZ G, MARTINEZ A. Sueño y Delirium en el paciente crítico. Acta Colombiana de Cuidado intensivo. [Internet] 2009 [citado junio 2012]; 9(1). Disponible en: http://www.anestesianet.com/actacci/ActaColCuidadoCriticoMar09.pdf (29) GONZALEZ AGUDELO Marco Antonio. Intervenciones para evitar la deprivación del sueño en los pacientes críticamente enfermos en la unidad de cuidados intensivos. Acta Colombiana de Cuidado intensivo. [Internet] 2010 [citado julio 2012]; 10(4). Disponible en: http://www.medintensiva.org/es/guia-practica-clinicabasada-videncia/articulo/S0210569113000855/

(30) COHEN Ana Maria. Calidad del sueño en los pacientes que ingresan en la unidad de cuidados intensivos. Maracaibo. [Internet] 2003 [citado mayo 2012]. Disponible en: http:/tesis.luz.edu.ve/tde_arquivos/17/TDE-2011-04-25T15:35:52Z833/Publico/cohen_ana_maria.pdf

(31) Siddigi N, Stockdale R, Britton AM, Holmes J. Intervenciones para la prevención del delirio en pacientes hospitalizados (Revisión Cochrane traducida). [Internet] 2008 [citado marzo 2011] Número 4. Disponible en: http://www.updatesoftware.com/BCP/BCPGetDocument.asp?DocumentID=CD005563

Recibido: 28 de octubre 2014; Aceptado: 16 de enero 2015

ISSN 1695-6141

() COPYRIGHT Servicio de Publicaciones - Universidad de Murcia 